\title{
A cluster of Listeria monocytogenes infections in hospitalised adults, Midlands, England, February 2011
}

N Coetzee (nic.cotzee@hpa.org.uk) ${ }^{1}$, V Laza-Stanca ${ }^{2}$, J M Orendi ${ }^{2}$, S Harvey ${ }^{3}$, N C Elviss ${ }^{4}$, K A Grant ${ }^{5}$

1. Health Protection Agency, West Midlands North, Stafford, United Kingdom

2. Department of Microbiology and Infection Control, University Hospital of North Staffordshire NHS Trust, Stoke-on-Trent, United Kingdom

3. Public Protection Division, Stoke on Trent City Council, Stoke-on-Trent, United Kingdom

4. Health Protection Agency, Food, Water and Environmental Microbiology Laboratory, Birmingham, United Kingdom

5. Health Protection Agency, Foodborne Pathogen Reference Unit, London, United Kingdom

Citation style for this article:

Coetzee N, Laza-Stanca V, Orendi JM, Harvey S, Elviss NC, Grant KA. A cluster of Listeria monocytogenes infections in hospitalised adults, Midlands, England, February 2011.

Euro Surveill. 2011;16(20):pii=19869. Available online: http://www.eurosurveillance.org/ViewArticle.aspx?Articleld=19869

Article published on 19 May 2011

Hospital-acquired listeriosis cases are not commonly reported but remain a significant public health problem. We report on three cases in patients with underlying conditions occurring during one week in February 2011. The cases had common exposure to pre-packed sandwiches and salads manufactured in compliance with regulations. Breaches in cold chain and shelf life controls at hospital level were identified as key contributing factors. Rigorous hospital food management systems remain important for patient safety.

\section{Case description and clinical diagnosis}

Listeria monocytogenes bacteraemia was confirmed in three patients admitted on 4, 5, and 6 February 2011 to a hospital in the Midlands region of England. Two were male and one was female. All lived in the same city served by the hospital but did not have any social links. Two cases were in the age range 50-59 years and one was older, over 80 years. All the three cases had underlying conditions which included malignancies and inflammatory bowel disease.

Cases were admitted in February 2011 to the same hospital where they had been hospitalised previously between 22 and 31 January 2011. Onset of symptoms leading to readmission of all three patients, ranged from 29 January to 3 February 2011, and these included fever, headache, confusion, abdominal pain and vomiting and L. monocytogenes was diagnosed in blood cultures three to four days after admission. All cases responded to antibiotic therapy with full recovery from infection.

\section{Investigation and control measures}

The 1,200-bed hospital is the only acute care facility in a district with approximately 500,000 inhabitants. A review of laboratory records for the preceding 12 months identified four unrelated (sporadic) communityacquired listeriosis cases. This background incidence rate is in keeping with national surveillance data, with 162,180 , and 139 non-pregnancy associated cases reported in 2008, 2009, and 2010 across England and Wales [1].
Following the identification of the three cases described above, an outbreak control team convened on 11 February 2011 to investigate the suspected outbreak and to advise on control measures.

Medical staff and management at the hospital were informed of the listeriosis cluster and the possibility of further cases. The hospital infection control team reinforced standard food avoidance advice for ready-to-eat foods commonly associated with listeriosis (such as pâté, smoked fish and mould ripened soft cheeses, or pre-packed sandwiches and salads) to patients with severe underlying conditions and/or on immunomodulating therapy, or pregnancy, and their families [2]. In addition, ward level food storage, distribution, and disposal practices were reviewed and staff reminded to follow existing protocols.

\section{Food history of patients}

Interviews with the three cases and their close relatives excluded animal contact and travel as relevant exposures. Food histories of the preceding four months did not identify common food preferences, consumption or purchasing while living at home. None had preference for ready-to-eat foods commonly associated with $L$. monocytogenes, nor were these present in their home refrigerators. The cases had attended the hospital outpatient department on various occasions between the two admissions, prior to disease onset, but had not eaten ready-to-eat foods from on-site shops. Hospital food was not served to patients attending the outpatient department.

The patients reported that during their hospital stays they had not eaten food (including ready-to-eat foods and sandwiches) from home or any of the eight privately-owned on-site visitor/staff canteens and shops. They had all consumed food provided by the hospital, and this had not been kept at room temperature but consumed immediately. The food histories were supplemented by a review of patient menu choice records 
kept by the hospital. The only risk factors (common food exposure) identified were pre-packed sandwiches and salads provided by the hospital during the common period of hospitalisation (22 to 31 January 2011). A wide variety of sandwiches and salads were eaten by all the cases, with no single sandwich or salad type being identified as unique common exposure. Salad types consumed included turkey, ham, cheese and coleslaw, and sandwich fillings included cheese, egg, ham, salmon, tuna, turkey, and tomato.

Isolates of L. monocytogenes from blood cultures of the three cases were identified as serogroup 4, and fluorescent amplified fragment length polymorphism (fAFLP) type V21. The isolates were thus indistinguishable by molecular typing, supporting a point source outbreak. In the absence of a common food exposure or source (no identified home-based common food exposure and no common food source that they used prior to the first hospital admission) the three cases were most likely exposed to contaminated food during their overlapping admission episode in January 2011. Based on this assumption, incubation periods were estimated to range from one-four days (minimum) to eight-13 days (maximum).

\section{Investigation of food suppliers}

An environmental health investigation confirmed that a single manufacturer supplied pre-packed sandwiches to the hospital for inpatient meals. Salad was prepared at the hospital central kitchen. At the hospital, samples for microbiological analysis were taken from ready-to-eat foods (pre-packed sandwiches, prepacked meats, cheddar cheese, cottage cheese used in on-site salad preparation, and completed salads), and kitchen drains. No L. monocytogenes was isolated from a total of 27 samples taken from this hospital between 10 and 24 February 2011.

A review of ready-to-eat food management practices at the hospital revealed that storage temperatures generally did not exceed $5^{\circ} \mathrm{C}$, but gaps in recordkeeping were found during evenings and weekends. Some instances were observed of ready-to-eat foods being accepted from the supplier at temperatures above $5^{\circ} \mathrm{C}$. Salad preparation in the hospital kitchens revealed lapses of the procedure for washing and disinfecting vegetables using chlorine. In addition, prepared salads were commonly given a two- or three-day shelf life rather than the recommended one day. Measures were taken to rectify these issues and food safety procedures are being updated at this hospital.

The eight privately owned on-site visitor/staff canteens and shops were inspected. Each was found to have different suppliers, and none of them supplied the same food as that given to inpatients in the hospital. Despite the fact that the three cases reported not to have obtained food from these eight outlets, 15 samples were taken of pre-packed sandwiches and salads as a precaution. L. monocytogenes serotype 4 ( $20 \mathrm{cfu} / \mathrm{g})$ was isolated from one ham and cheddar cheese sandwich but the fAFLP type differed from the isolates of the three cases.

A full production hygiene investigation (focused on sandwich and salad component production) of the manufacturer supplying food for hospital inpatients was undertaken by local environmental officers. There was a fully documented hazard analysis and control system in place and the quality assurance programme included daily microbiological testing of sandwiches for indicator organisms at three days after production (end of shelf life), with enumeration testing for Listeria spp., including L. monocytogenes, approximately every ten days.

For the five months prior to 20 December 2010, none of 38 samples exceeded $10 \mathrm{cfu} / \mathrm{g}$ for Listeria spp. Due to adverse winter weather conditions routine sampling had ceased from 21 December 2010, to be resumed only after the detection of the three listeriosis cases early in February 2011. Based on hazard analysis control system documentation, no breach of production quality processes was detected during this period. Further independent sampling by environmental health officers on 23 February 2011 did not detect $L$. monocytogenes in ten sandwich samples and 15 environmental (food production sites and drains) samples. From March 2011, the company revised their microbiological sampling plan (including sampling sandwiches on day of production) and are now using both enumeration and enrichment techniques in $L$. monocytogenes detection. To date, no further L. monocytogenes isolates in sandwich samples have been detected from the supplier.

\section{Discussion and conclusion}

Detailed investigations identified the consumption of hospital supplied sandwiches and/or salads during the last ten days in January 2011 to be a likely risk factor for infection with L. monocytogenes. The cluster is unlikely to be due to a chance occurrence, as cases occurred close together in excess of background incidence, had overlapping hospital stay, and isolates were indistinguishable by fAFLP typing. Microbiological evidence that hospital supplied food was the source of infection could not be established.

The sandwich producer follows the British Sandwich Association target microbiological standard in finished products (at end of shelf life) of Listeria spp. at $<10$ $\mathrm{cfu} / \mathrm{g}$ which is compliant with European Commission (EC) regulations $[3,4]$. Whilst the detection of $<10 \mathrm{cfu} / \mathrm{g}$ of L. monocytogenes in sandwiches and salads supplied to patients provides some assurance, sample numbers were low and taken more than ten days after cases were likely to have been exposed. In addition, the break in sampling by the producer before and during the case exposure period coupled with significant cold chain breaches and extended salad shelf life at the hospital preceded the cases. Two extensive United Kingdom (UK)-wide microbiological surveys of 
sandwich quality served at hospitals and healthcare institutions reported $2.7-3.1 \%$ of samples containing L. monocytogenes [5]. In both studies, the presence of Listeria spp. and L. monocytogenes was associated with sandwiches produced outside the hospital, and where storage above $8^{\circ} \mathrm{C}$ had occurred.

In our experience sandwiches are commonly consumed by all patients in the study hospital, as well as most hospitals across the UK $[6,7]$. Even low levels of $L$. monocytogenes in sandwiches and ready-to-eat foods pose a risk to certain immunocompromised patients and pregnant women. The vast majority of sandwiches are safe, and hospital incidents and outbreaks of listeriosis are relatively infrequent, with six outbreaks reported in England and Wales from 1999 to 2008 [7]. However, listeriosis is a serious disease in compromised patients, and despite low numbers it remains a significant patient safety concern. Leading investigators have therefore recommended that food served to hospital patients to be free from potential pathogens, including L. monocytogenes [5-7].

Although this hospital food manufacturer supplies food to many other hospitals in the UK, no further laboratory confirmed cases of listeriosis of the same fAFLP type (V21) were identified in the UK more than 10 weeks after the cluster was detected and there were no other outbreaks of listeriosis or sporadic cases of this unique fAFLP type. Even though the ready-to-eat foods in this study were manufactured in accordance with the European Union regulations [4], it is possible that lack of temperature and shelflife controls at the study hospital were key factors leading to increases in listeriosis and infection in vulnerable patients.

The investigation of hospital-based $L$. monocytogenes outbreaks is notoriously difficult due to low attack rates, incomplete case ascertainment, food histories spanning long periods, and food samples often being negative or taken long after the exposure time [7]. Such outbreaks are likely to be underreported, with publication bias towards larger outbreaks confirming microbiological food exposures. In order to develop appropriate future control strategies for this ongoing public health problem, we recommend that investigators make every effort to report and publish the full spectrum of hospital associated L. monocytogenes clusters and outbreaks.

\section{Acknowledgments}

We would like to thank the hospital infection control team, environmental health officers (Newcastle-under-Lyme Borough Council, Stoke-on-Trent City Council, and City \& County of Swansea), Jeremy Hawker (HPA West Midlands), Public Health Wales, and Corinne Amar (HPA Foodborne Pathogen Reference Unit London) for fAFLP analysis of Listeria monocytogenes isolates.
References

1. Health Protection Agency (HPA). Listeria monocytogenes. Human cases in England and Wales reported to the HPA Centre for Infections, 1983-2010. London:HPA. 10 Mar 2011. Available from: http://www.hpa.org.uk/Topics/InfectiousDiseases/ InfectionsAZ/Listeria/EpidemiologicalData/ listeCaseReports19832010/

2. NHS choices. Listeriosis. [Accessed 10 May 2011]. Available from: http://www.nhs.uk/Conditions/Listeriosis/Pages/ Introduction.aspx

3. British Sandwich Association. Code of Practice and Minimum Standards for Sandwich Manufacturers (Producers) (updated August 2007). [Accessed 9 May 2011]. Available from: http:// www.sandwichesonline.org.uk/about_the_bsa/manufacturer_ code_of_practice.shtml

4. European Commission (EC). Commission Regulation (EC) No $1441 / 2007$ of 5 December 2007 amending Regulation (EC) No 2073/2005 on microbiological criteria for foodstuffs. EC. 7 Dec 2007. Available from: http://eur-lex.europa.eu/LexUriServ/ LexUriServ.do?uri=0J:L:2007:322:0012:0029:EN:PDF

5. Little CL, Barrett NJ, Grant K, McLauchlin J. Microbiological safety of sandwiches from hospitals and other health care establishments in the United Kingdom with a focus on Listeria monocytogenes and other Listeria species. J Food Prot. 2008;71(2):309-18.

6. Shetty A, McLauchlin J, Grant K, O'Brien D, Howard T, Davies EM. Outbreak of Listeria monocytogenes in an oncology unit associated with sandwiches consumed in hospital. J Hospt Infect. 2009;72(4):332-6.

7. Health Protection Agency (HPA). Human listeriosis linked to hospital sandwiches: implications for procurement and storage. London:HPA. Health Protection Report. 2008:2(35). Available from: http://www.hpa.org.uk/hpr/archives/2008/ news $3508 . \mathrm{htm}$. 